\title{
Article \\ Synthesis of Single-Crystal Graphene on Copper Foils Using a Low-Nucleation-Density Region in a Quartz Boat
}

\author{
Kaiqiang Yang ${ }^{1,2}$, Jianlong Liu ${ }^{1}$, Ruirui Jiang ${ }^{1}$, Yubin Gong ${ }^{1}$, Baoqing Zeng ${ }^{1, * \mathbb{C}}$, Zichuan $\mathrm{Yi}^{2}$, Qingguo Gao ${ }^{2}$, \\ Jianjun Yang ${ }^{2}$, Feng $\mathrm{Chi}^{2}$ and Liming Liu ${ }^{2, *}$ \\ 1 School of Electronic Science and Engineering, University of Electronic Science and Technology of China, \\ Chengdu 610054, China; 201811022515@std.uestc.edu.cn (K.Y.); liujianlong@uestc.edu.cn (J.L.); \\ 201711040120@std.uestc.edu.cn (R.J.); ybgong@uestc.edu.cn (Y.G.) \\ 2 Zhongshan Branch of State Key Laboratory of Electronic Thin Films and Integrated Devices, \\ Zhongshan Institute, University of Electronic Science and Technology of China, Zhongshan 528402, China; \\ yizichuan@zsc.edu.cn (Z.Y.); gqg@hust.edu.cn (Q.G.); sdyman@uestc.edu.cn (J.Y.); chifeng@semi.ac.cn (F.C.) \\ * Correspondence: bqzeng@uestc.edu.cn (B.Z.); limingliu@uestc.edu.cn (L.L.)
}

Citation: Yang, K.; Liu, J.; Jiang, R.; Gong, Y.; Zeng, B.; Yi, Z.; Gao, Q.;

Yang, J.; Chi, F.; Liu, L. Synthesis of Single-Crystal Graphene on Copper Foils Using a Low-NucleationDensity Region in a Quartz Boat. Micromachines 2021, 12, 1236. https:// doi.org $/ 10.3390 / \mathrm{mi} 12101236$

Academic Editor: Ha Duong Ngo

Received: 2 September 2021

Accepted: 5 October 2021

Published: 12 October 2021

Publisher's Note: MDPI stays neutral with regard to jurisdictional claims in published maps and institutional affiliations.

Copyright: $\odot 2021$ by the authors. Licensee MDPI, Basel, Switzerland. This article is an open access article distributed under the terms and conditions of the Creative Commons Attribution (CC BY) license (https:// creativecommons.org/licenses/by/ $4.0 /)$.

\begin{abstract}
The nucleation of graphene at different locations in the quartz boat was studied, and the lowest nucleation density of graphene in the quartz boat was found. The nucleation density of graphene is the lowest at the bottom of the quartz boat near the gas inlet side. Based on the above results, a simple and reproducible way is proposed to significantly suppress the nucleation density of graphene on the copper foil during the chemical vapor deposition process. Placing the copper foil with an area of $1.3 \mathrm{~cm} \times 1 \mathrm{~cm}$ in the middle of the bottom of the quartz boat or further back, and placing two copper pockets in front of the copper foil, an ultra-low nucleation density of $\sim 42$ nucleus $/ \mathrm{cm}^{2}$ was achieved on the back of the copper foil. Single-crystal monolayer graphene with a lateral size of $800 \mu \mathrm{m}$ can be grown on the back of copper foils after $60 \mathrm{~min}$ of growth. Raman spectroscopy revealed the single-crystal graphene to be in uniform monolayers with a low D-band intensity.
\end{abstract}

Keywords: single-crystal graphene; nucleation density; quartz boat; copper foils; copper pockets

\section{Introduction}

Graphene is composed of two-dimensional carbon atoms assembled in a hexagonal structure, akin to a honeycomb, and has exceptional chemical stability, superior mechanical stability, and very high thermal conductivities and electronic conductivities [1-3]. Because of the above excellent properties, graphene has potential applications in ultra-high-speed electronics [4], flexible transparent conductive films [5,6], solar cells [6], separation membranes [7], and transmission electron microscopy (TEM) imaging [8]. Graphene was first isolated by mechanical exfoliation from Highly Oriented Pyrolytic Graphite (HOPG) [1]. Although various methods have been used to grow graphene on a solid surface such as HOPG, electrochemical exfoliation, epitaxy growth, and chemical reduction of graphene oxide [9], chemical vapor deposition (CVD) on metal foils is believed to be a promising method to produce large-area mono- or few-layer graphene [10]. However, a polycrystalline structure and a high density of domain/grain boundaries in typical graphene grown by CVD on metal foils cause variable electronic properties in different areas and hinder the applications of graphene in devices [11,12]. Graphene grain boundaries (GGBs), which form in regions where graphene domains with different orientations merge, are observed as line defects in graphene film and result in the degradation of the thermal, electrical, and chemical properties of graphene [13-18]. Accordingly, it is necessary to study the growth and preparation of single-crystal graphene. For example, the electrical performance of graphene is degraded by the additional scattering centers induced by GGBs, causing a reduction in the carrier mobility and conductivity of graphene [19]. 
It is still a challenge to grow large-size single-crystal graphene. There are two ideas to improve the size of single-crystal graphene, which are the single-seed method and the multi-seed method [20]. The key of the single-seed method is to control the nucleation density of graphene. Maintaining lower surface roughness of the substrate and reducing the supply of carbon are two main methods to reduce the nucleation density of graphene. One classic method is the copper pocket method [21], which is used to explore the distribution of methane partial pressure in a quartz boat in our work. The multi-seed method requires the preparation of special substrates. The orientation dependence of graphene on the substrate surfaces, which is vital to the aligned nucleation of graphene islands, is mainly determined by the interaction and the lattice-matching degree between graphene and the underlying substrates. To date, the epitaxial growth of graphene has been reported on the surfaces of some special substrates such as $\mathrm{Cu}(111), \mathrm{Ni}(111)$ [22], $\mathrm{Au}(111)$ [23], $\mathrm{Pt}(111)$ [24], and $\mathrm{CuNi}$ alloy $[25,26]$. Among these, $\mathrm{Cu}(111)$ plane is highly advantageous for the aligned nucleation and growth of graphene domains, as its hexagonal lattice symmetry matches the honeycomb lattice of graphene well (lattice mismatch $\approx 4 \%$ ), thereby enabling the epitaxial growth of graphene, upon which the graphene islands can demonstrate simultaneous growth and seamless merging [27-29].

In this paper, the nucleation of graphene at different locations in the quartz boat isexplored through three groups of comparative experiments. Based on the results of these experiments, we report a simple and reproducible way to significantly suppress the nucleation density by placing the copper foil with an area of $1.3 \mathrm{~cm} \times 1.0 \mathrm{~cm}$ in the middle of the bottom of the quartz boat, or further back, and placing two copper pockets in front of the copper foil. Nucleation density of graphene on the back of copper foils can be significantly reduced and an ultra-low nucleation density of $\sim 42$ nucleus $/ \mathrm{cm}^{2}$ was achieved. After increasing the growth time to $60 \mathrm{~min}$, single-crystal monolayer graphene with a lateral size of $800 \mu \mathrm{m}$ can be grown on the back of copper foils under the optimized growth condition. In addition, the Raman study indicates that the single-crystal graphene synthetized by our method has a very low D-band intensity on the silicon dioxide grown on Si wafers, which is of high quality.

\section{Materials and Methods}

\subsection{Synthesis of Graphene}

Graphene was synthesized by the low-pressure CVD in a tube furnace. The copper foils were folded into copper pockets, and a Cu pocket was made by first bending a copper foil (99.8\%, Alfa Aesar, Shanghai, China, annealed, uncoated, \#46365) and then crimping or pressing the three open edges carefully by using metal tweezers [30]. The copper pockets were placed flat in the quartz boat, which was located in the center of the quartz tube of a CVD system. The whole interior of the quartz boat is divided into four regions. These four regions are referred to as location 1 to location 4 , respectively. Horizontally, the locations of the copper pockets in the quartz boat are shown in Figure 1a. Vertically, the locations of the copper pockets in the quartz boat are shown in Figure $1 \mathrm{~b}, \mathrm{~d}$ is the distance from the copper pocket to the bottom of the quartz boat. Three groups of experiments were completed for exploring the nucleation of graphene in different regions of the quartz boat. Two copper pockets placed in different locations in the quartz boat were added to the CVD system in each experiment. In the first group of experiments, two copper pockets were located in location 2 and location 3 of the quartz boat, respectively. In the second group of experiments, two copper pockets were located in location 1 and location 4 of the quartz boat, respectively. In the third group of experiments, two copper pockets were located in location 2 and location 4 of the quartz boat, respectively. The same parameters were used in the three experiments: furnace temperature $1030^{\circ} \mathrm{C} \mathrm{Ar:} \mathrm{H}_{2}: \mathrm{CH}_{4}=50: 50: 0.5$ (in the unit of sccm), and growth time of $45 \mathrm{~min}$. After studying the nucleation of graphene at different locations in the quartz boat, a new growth mode was used. A piece of copper foil with an area of $1.3 \mathrm{~cm} \times 1.0 \mathrm{~cm}$ was prepared and then placed at the middle position of the bottom of the quartz boat. The distance between the copper foil and the bottom of 
the quartz boat was $0.5 \mathrm{~cm}$, and the center of the copper foil was about $4 \mathrm{~cm}$ away from the tail of quartz boat (the quartz boat was $10 \mathrm{~cm}$ long, $4 \mathrm{~cm}$ wide, and $2 \mathrm{~cm}$ high). At the same time, two copper pockets were placed in front of the copper foil in order to further reduce the methane flow at the bottom of the quartz boat, as shown in Figure 6a. Finally, the quartz boat with copper foils was placed into the quartz tube and the growth test was carried out in the center of the heating furnace. The growth parameters were as follows: furnace temperature $1030{ }^{\circ} \mathrm{C}, \mathrm{Ar}: \mathrm{H}_{2}: \mathrm{CH}_{4}=50: 50: 0.5$ (in the unit of sccm), and growth time of $60 \mathrm{~min}$. Each group of experiments was repeated multiple times, until the results were stable.

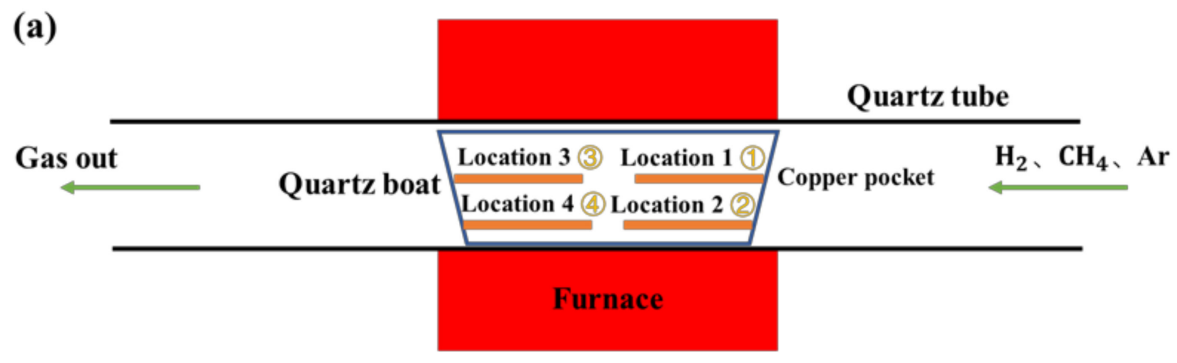

(b)

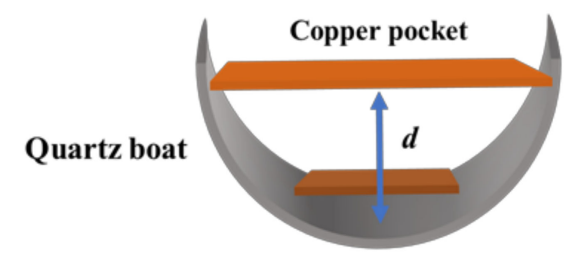

Figure 1. (a) Schematic illustrations of the distribution of four regions in the quartz boat in the transverse direction and the CVD system. (b) Schematic illustrations of the copper pockets placed in the quartz boat.

\subsection{Transfer of Graphene}

A thin film of polymethyl methacrylate (PMMA) (HF-kejing, Hefei, China, $6 \mathrm{wt} . \%$ in anisole) was spin-coated onto the graphene/ $\mathrm{Cu}(600 \mathrm{r} / \mathrm{min}$ for $6 \mathrm{~s}$ then $3000 \mathrm{r} / \mathrm{min}$ for $40 \mathrm{~s}$ ). The etching of $\mathrm{Cu}$ usually takes $3 \mathrm{~h}$. Then, the PMMA/graphene/ $\mathrm{Cu}$ thin film was placed into $30 \mathrm{mg} / \mathrm{mL}$ ammonium persulfate $\left(\left(\mathrm{NH}_{4}\right)_{2} \mathrm{~S}_{2} \mathrm{O}_{8}\right)$ aqueous solution (Aladdin, Shanghai, China, purity of $98 \%$, AR). After etching, the PMMA/graphene was rinsed in DI water several times. The PMMA/graphene was dried in a desiccator and then placed on the $\mathrm{SiO}_{2} / \mathrm{Si}$ substrate. The PMMA layer was dissolved using hot acetone (Guangzhou, China, purity of $99.5 \%$, AR).

\subsection{Characterization}

After finishing the growth of graphene, the copper pockets and copper foils were opened to copper foils, then the copper foils were placed on the hot table at $200{ }^{\circ} \mathrm{C}$ for $5 \mathrm{~min}$. The oxidized surfaces of copper foils were observed under the optical microscope. Due to the effect of the oxidation resistance of graphene [31], the areas covered by graphene were not oxidized, and the areas not covered with graphene were oxidized to copper oxide. The areas covered by graphene were brighter than other areas. Therefore, we could directly observe the shape and size of single-crystal graphene on copper foils by optical microscope. The diameter and nucleation density of single-crystal graphene under different growth conditions are the average values of repeated experiments. Scanning electron microscopy (TESCAN, Brno, Czech Republic) was also used to characterize the morphology of graphene. Raman spectroscopy (FuXiang, Shanghai, China) was used to characterize the number and quality of graphene layers. 


\section{Results and Discussion}

In the first group of experiments, the copper pockets were placed as shown in Figure 2a. Location 2 and location 3 are described as the bottom of the quartz boat near the gas inlet side and the top of the quartz boat near the gas outlet side in the following, respectively. As shown in Figure 2b,c, the nucleation density of graphene on the inner surface of the copper pockets at location 2 was lower than that on the inner surface of the copper pockets at location 3 after growing for $45 \mathrm{~min}$. The inner surface of the copper pockets at location 2 was not completely covered by graphene, the distribution of nucleation points of graphene was scattered, and there were many places without graphene. Figure $2 \mathrm{~d}$ is the optical micrograph of the inner surface of copper pockets at location 2 under 100-times magnification. Single-crystal graphene grains could be observed under the microscope. The shape of single-crystal graphene was approximately hexagonal, and the average diameter of the hexagonal grains was $335 \mu \mathrm{m}$. Figure $2 \mathrm{e}$ is the optical micrograph of the inner surface of copper pockets at location 3 under 100-times magnification. Single-crystal graphene was not observed, and the graphene regions were combined with each other; only a few areas were covered with copper oxide (dark red area). Here, we use the reported graphene visualization methods [31], where the graphene/copper sample is oxidized in air and results in a reflective contrast between the oxidized copper in the exposed regions and the unexposed copper covered by graphene. Through the above observation, we found that the nucleation density of graphene on the bottom of the quartz boat near the gas inlet side was lower than that in the top of the quartz boat near the gas outlet side with the copper pockets method.

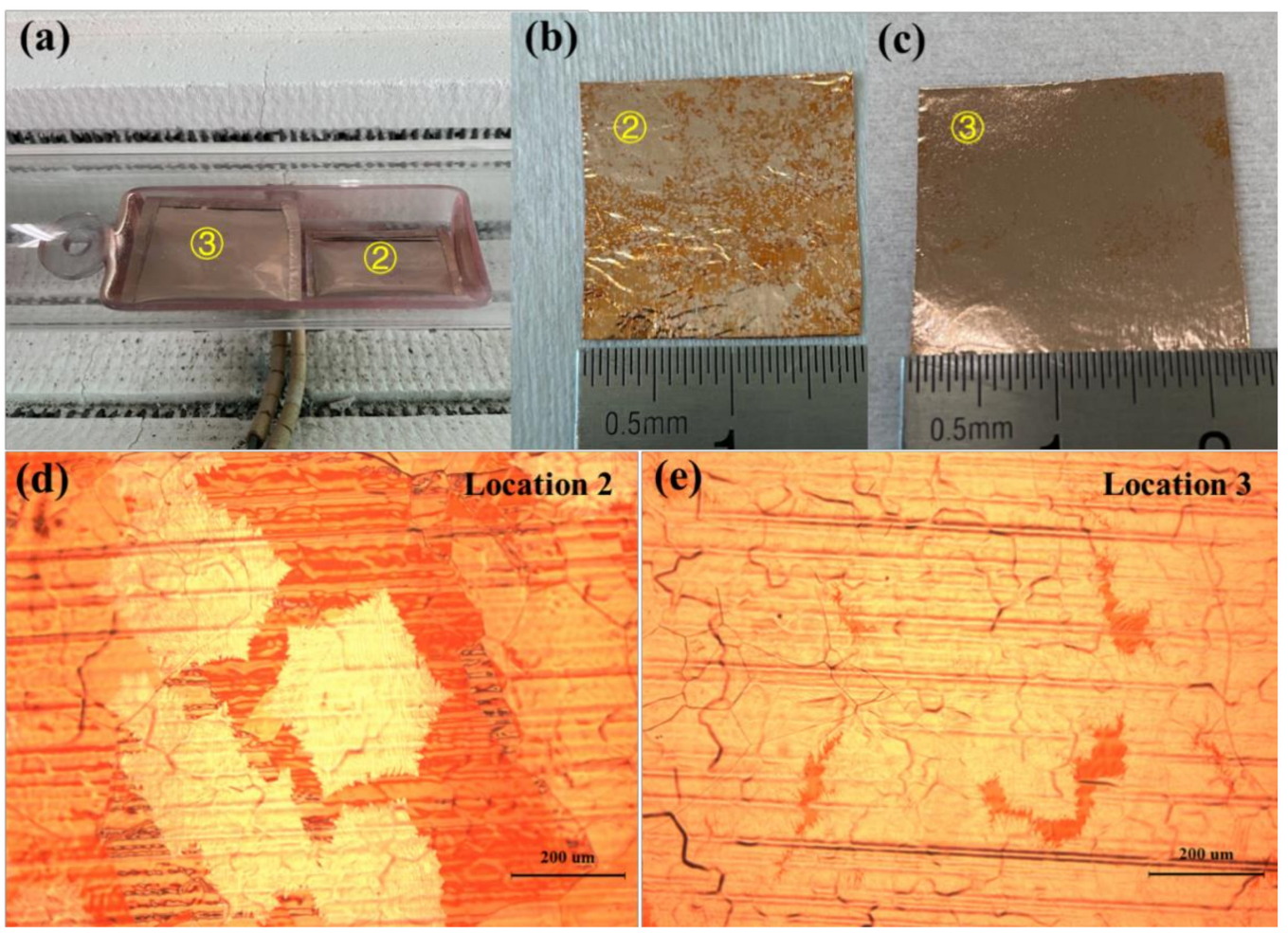

Figure 2. (a) Optical picture of the two copper pockets placed in the quartz boat in the first experiment. (b,c) Optical picture of the inner surfaces of the copper pockets at location 2 and location 3 after oxidation treatment. (d,e) Optical micrograph of the inner surfaces of the copper pockets at location 2 and location 3 under 100-times magnification.

In the second group of experiments, the copper pockets were placed as shown in Figure 3a. Location 4 and location 1 are described as the bottom of the quartz boat near the gas outlet side and the top of the quartz boat near the gas inlet side in the following, respectively. As shown in Figure 3b,c, the inner surface of the copper pockets at location 1 
was completely covered by graphene, but there were still some areas on the inner surface of the copper pocket at location 4 which were not covered by graphene. Figure $3 \mathrm{~d}$ is the optical micrograph of the inner surface of the copper pockets at location 1 under 100-times magnification. Single-crystal graphene was not observed, and the graphene regions were combined with each other; the substrate was completely covered with graphene. Figure $3 \mathrm{e}$ is the optical micrograph of the inner surface of the copper pockets at location 4 under 100-times magnification. Single-crystal graphene grains could be clearly observed under the microscope. The regions of single-crystal graphene were independent, with an average size of $215 \mu \mathrm{m}$. We found that the nucleation density of graphene in the bottom of the quartz boat near the gas outlet side was lower than that in the top of the quartz boat near the gas inlet side from the second group of experiments.

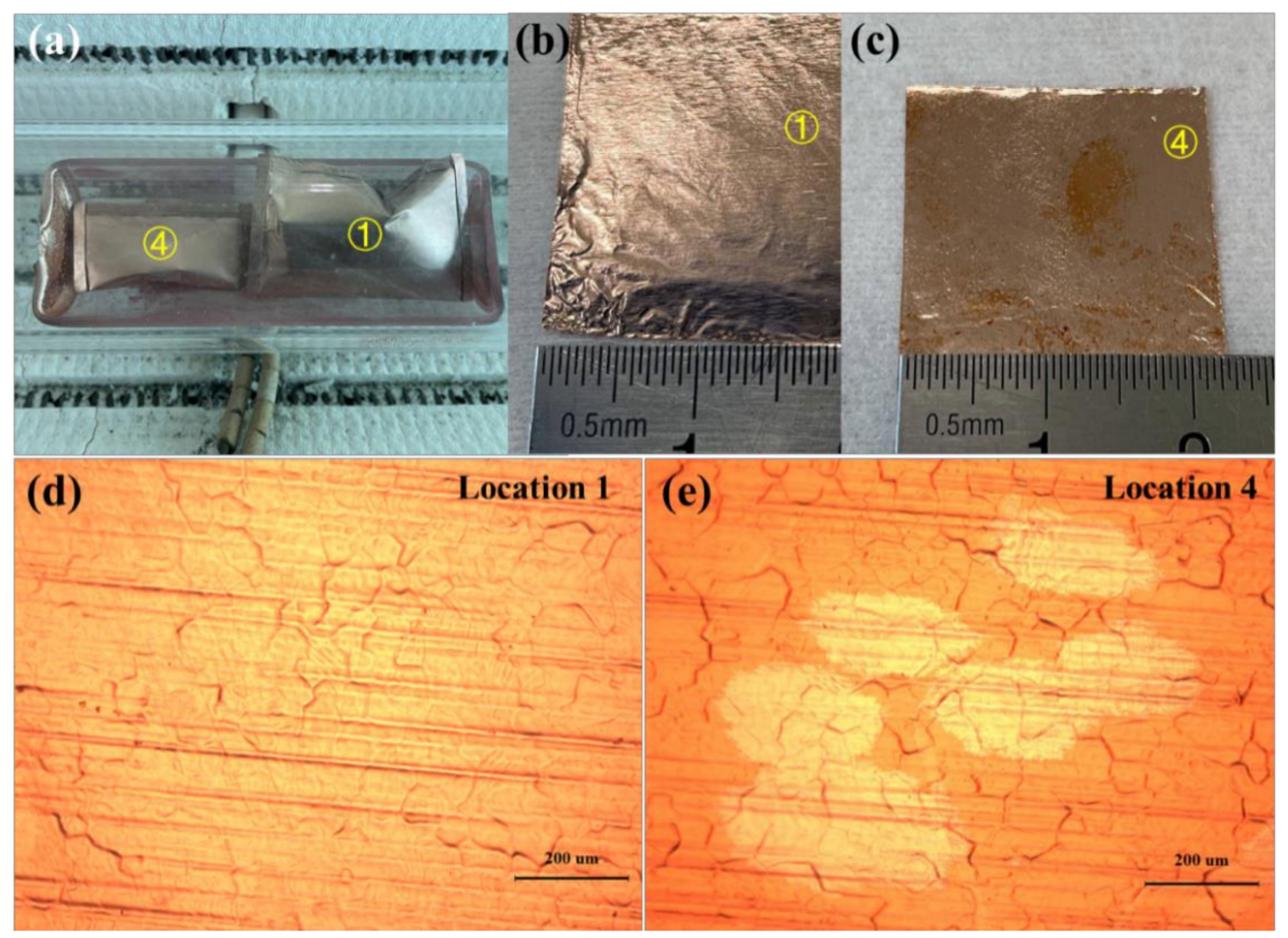

Figure 3. (a) Optical picture of the two copper pockets placed in the quartz boat in the second group of experiments. $(\mathbf{b}, \mathbf{c})$ Optical picture of the inner surfaces of the copper pockets at location 1 and location 4 after oxidation treatment. (d,e) Optical micrograph of the inner surfaces of the copper pockets at location 1 and location 4 under 100-times magnification.

In the third group of experiments, two copper pockets were placed as shown in Figure 4a. As shown in Figure $4 b, c$, the inner surfaces of the copper pockets at location 2 and location 4 were not completely covered by graphene, and the distribution of the graphene regions was scattered. Comparing Figure $4 b, c$, we also find that the area not covered by graphene on the inner surface of the copper pockets at location 2 was larger than that at location 4 , and the nucleation density of graphene on the inner surface of the copper pockets at location 2 was lower than that at location 4 . Figure $4 \mathrm{~d}$ is the optical micrograph of the inner surface of the copper pockets at location 2 under 100-times magnification. Single-crystal graphene grains could be observed under the microscope. The shape of single-crystal graphene was approximately hexagonal, and the average diameter of the hexagonal grains was $467 \mu \mathrm{m}$. Figure $4 \mathrm{e}$ is the optical micrograph of the inner surface of the copper pockets at location 4 under 100-times magnification. Single-crystal graphene grains could be observed under the microscope. The average size of single-crystal graphene was $221 \mu \mathrm{m}$. Summing up the third group of experiments, we found that the nucleation density of graphene in the bottom of the quartz boat near the gas inlet side was lower than that in the bottom of the quartz boat near the gas outlet side. 


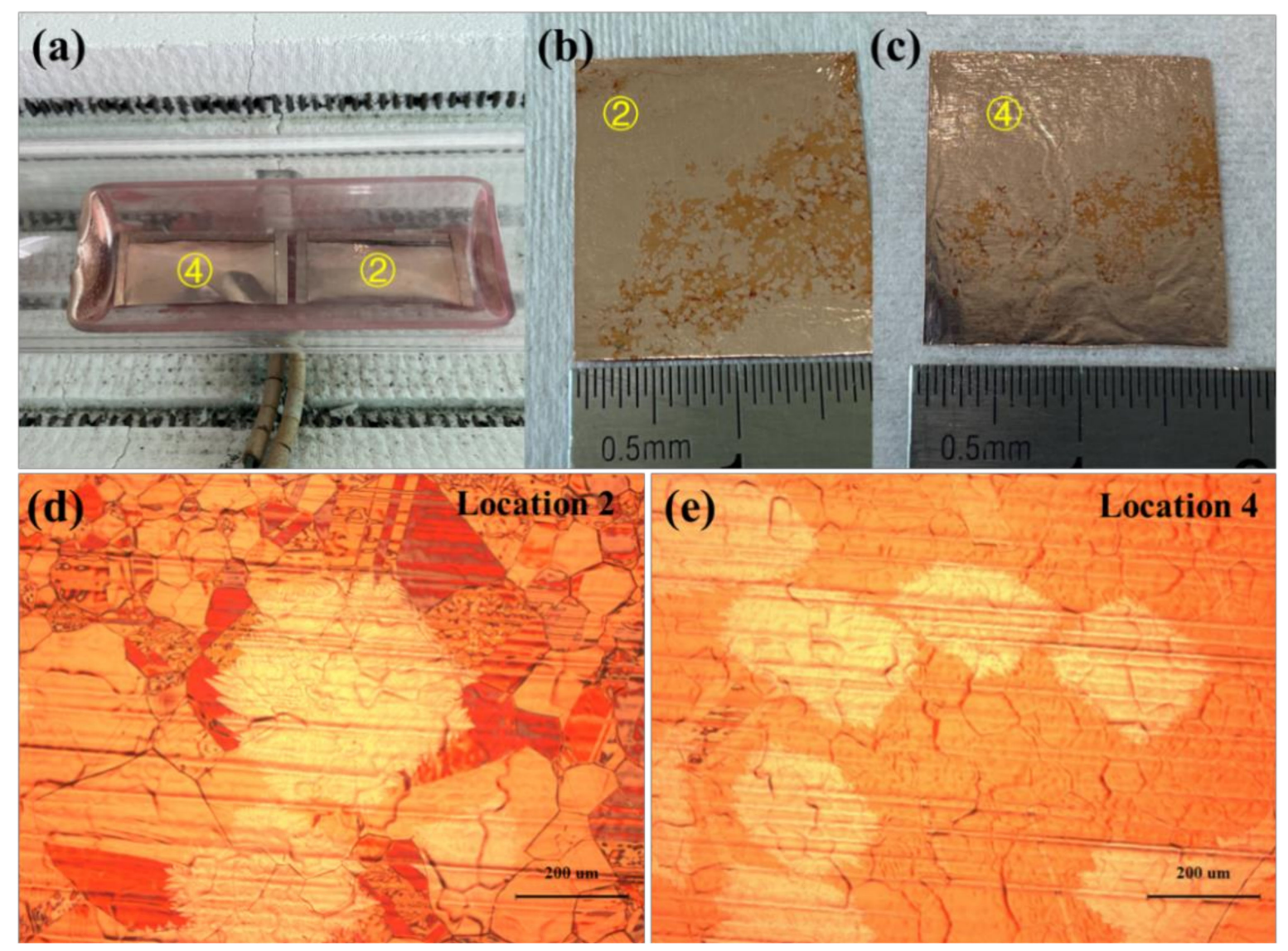

Figure 4. (a) Optical picture of the two copper pockets placed in the quartz boat in the third group of experiments. $(\mathbf{b}, \mathbf{c})$ Optical picture of the inner surfaces of the copper pockets at location 2 and location 4 after oxidation treatment. (d,e) Optical micrograph of the inner surfaces of the copper pockets at location 2 and location 4 under 100-times magnification.

In our experiments, we also observed that the single-crystal graphene can grow on the back of the outer surfaces of the copper pockets (the side near the bottom of the quartz boat) and the nucleation density of graphene was very low. There were only about 102 nucleation sites of graphene in the area of $3.5 \mathrm{~cm} \times 2 \mathrm{~cm}$, and the nucleation density of graphene is 27 nucleus $/ \mathrm{cm}^{2}$. Figure $5 \mathrm{a}$ is an optical picture of the back of the outer surface of the copper pockets at location 2 . The nucleation points of graphene were sparsely distributed on the copper foil. Figure $5 \mathrm{c}$ is the optical micrograph of the back of the outer surface of the copper pockets at location 2 under 50-times magnification. Single-crystal graphene grains could be observed under the microscope. The shape of single-crystal graphene was approximately hexagonal, and the average diameter of the hexagonal grains was $398 \mu \mathrm{m}$. Figure $5 \mathrm{~b}$ is an optical picture of the back of the outer surface of the copper pockets at location 4 . The lighter areas are graphene grains (the color contrast between graphene and oxidized substrate is not obvious). The area covered by graphene was larger than that at location 2. The nucleation density of graphene was also higher than that at location 2. Figure $5 \mathrm{~d}$ is the optical micrograph of the back of the outer surface of the copper pockets at location 2 under 50-times magnification. Single-crystal graphene grains could also be observed under the microscope. The diameter of single-crystal graphene was in the range of 50-360 $\mu \mathrm{m}$. In this group of experiments, the nucleation density of graphene in the bottom of the quartz boat near the gas inlet side was lower than that in the bottom of the quartz boat near the gas outlet side, which also supports the conclusion of the third group of experiments. 


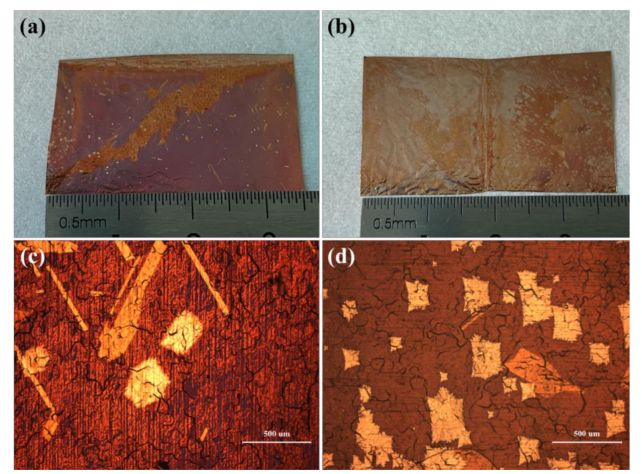

Figure 5. (a,b) Optical picture of the back of the copper pockets (outer surfaces) at location 2 and location 4 after oxidation treatment. (c,d) Optical micrograph of the back of the copper pockets (outer surfaces) at location 2 and location 4 under 50 -times magnification.

The above four groups of experiments were carried out multiple times in each group. The distribution of the nucleation density of graphene at four locations in the quartz boat was obtained after stable results appeared. We found that the nucleation density of graphene at the bottom of the quartz boat (location 2 or location 4) was lower than that at the top (location 1 or location 3), and the closer to the gas inlet side (location 1 or location 2), the lower the nucleation density of the graphene in the copper pockets method. In the copper pockets method, the growth of single-crystal graphene depends on the smooth inner surface of the copper pockets during the growth process. The smoothness of the inner surface is created by the dynamic balance of the evaporation and deposition of copper inside copper pockets [32]. In addition, the closed structure of copper pockets also makes the partial pressure of methane in the copper pockets very low. Low methane partial pressure can effectively reduce the nucleation density of graphene, which makes it possible to prepare large-size single-crystal graphene [21]. Therefore, the different nucleation density of graphene in the quartz boat should be related to the partial pressure of methane in the quartz boat. In the growth stage, the partial pressure of methane at the bottom was lower than that at the top, and that at the location near the gas inlet side was lower than that near the gas outlet in the quartz boat.

Based on the above results, we proposed a simple and reproducible way to synthesize single-crystal graphene. A copper foil with an area of $1.3 \mathrm{~cm} \times 1.0 \mathrm{~cm}$ was placed on the middle of the bottom of the quartz boat, or further back, and two copper pockets were placed in front of the copper foil, as shown in Figure 6a. The copper foils being placed in the middle of the bottom of the quartz boat, or further back, was based on our previous experiments, where the partial pressure of methane was low and the nucleation density of graphene was low. Two copper pockets were placed in front of the copper foils to further reduce the methane rate at the bottom of the quartz boat. The copper pockets played a role in shielding, so as to reduce the partial pressure of methane at the bottom of the quartz boat, and the nucleation density of graphene on the copper foils was also lower. In many experiments, we found that as long as the copper foils that were cut to about $1 \mathrm{~cm}^{2}$ (at this time, the back of copper foils were about $0.5 \mathrm{~cm}$ away from the bottom of the quartz boat) were placed at the bottom of quartz boat, and two copper pockets were in front of this, single-crystal graphene could be stably obtained on the surfaces of copper foils. Figure $6 \mathrm{~b}$ is an optical picture of the back of the copper foil with single-crystal graphene. After $45 \mathrm{~min}$ of growth, there were 58 nucleation points of graphene on the back of the copper foil (the side facing the bottom of the quartz boat). The nucleation density of graphene was about 42 nucleus $/ \mathrm{cm}^{2}$ under this condition. Figure $6 \mathrm{c}$ is the optical micrograph of the back of the copper foil under 50-times magnification. Figure $6 \mathrm{~d}$ is the optical micrograph of the back of the copper foil under 100-times magnification. Single-crystal graphene grains could be observed under the microscope. The average diameter of single-crystal graphene is $429 \mu \mathrm{m}$. 

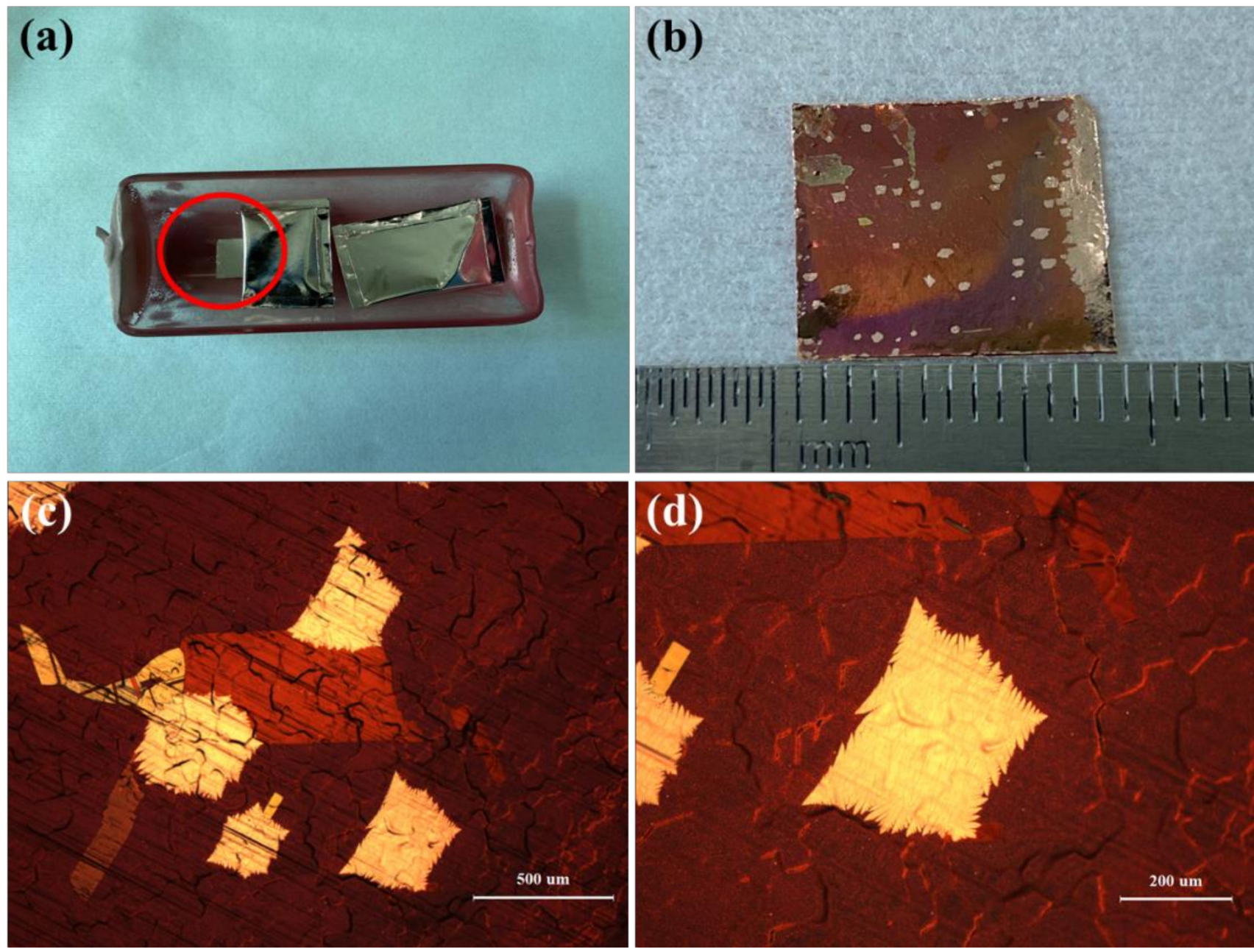

Figure 6. (a) Optical picture of the copper foil placed in the quartz boat in the experiment. (b) Optical picture of the back of the copper foil with single-crystal graphene. (c) Optical micrograph of single-crystal graphene on the back of the copper foil under 50-times magnification. (d) Optical micrograph of single-crystal graphene on the back of the copper foil under 100-times magnification.

By placing copper foils on the bottom of the quartz boat (two copper pockets were placed in front of the copper foils, as shown in Figure 6a), a low nucleation density of graphene was obtained at the back of the copper foil. When growth time was increased to $60 \mathrm{~min}$, single-crystal graphene of a diameter about $800 \mu \mathrm{m}$ was obtained, under other unchanged growth conditions. Figure $7 \mathrm{a}$ is the optical micrograph of single-crystal graphene on the back of the copper foil under 100-times magnification. The average diameter of single-crystal graphene was about $800 \mu \mathrm{m}$. Figure $7 \mathrm{~b}$ is the SEM image of graphene domains on the copper foil, and the edge of single-crystal graphene presents a fractal structure resembling a snowflake. Single-crystal graphene domains on the copper foils were transferred onto dielectric substrates such as silicon dioxide, using a poly(methyl methacrylate) (PMMA)-assisted method for Raman characterizations. Figure 7c is the optical micrograph of single-crystal graphene on silicon dioxide grown on Si wafers (the thickness of silicon dioxide is $300 \mathrm{~nm}$ ) under 100-times magnification. Figure 7d shows the Raman spectra of the single-crystal graphene transferred onto the silicon dioxide grown on $\mathrm{Si}$ wafers. The $\mathrm{I}_{2 \mathrm{D}} / \mathrm{I}_{\mathrm{G}}$ intensity ratio was $\sim 1.2$, and there was no obvious $\mathrm{D}$ peak. High-quality graphene was obtained by our growth and transfer method. 

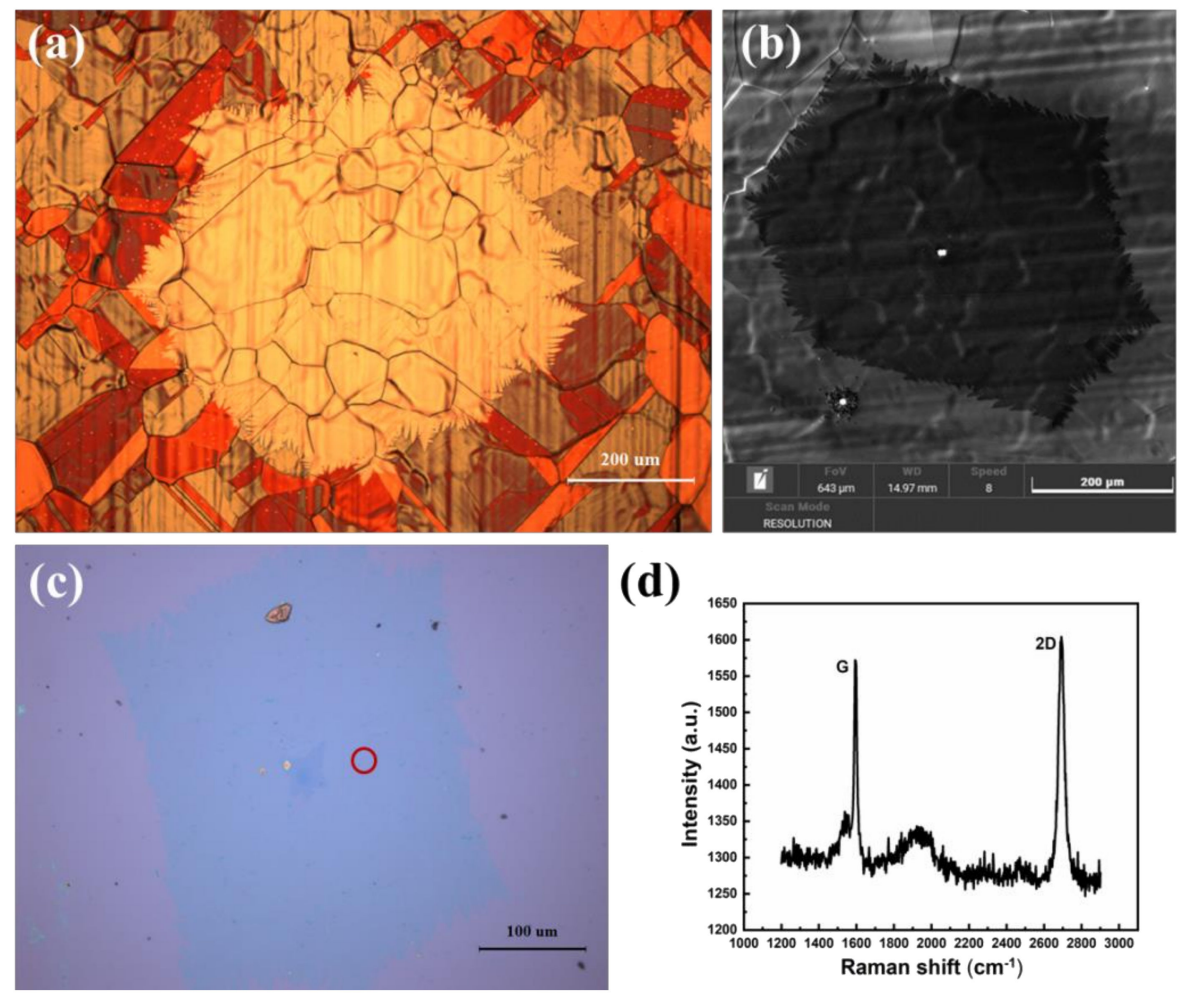

(d)

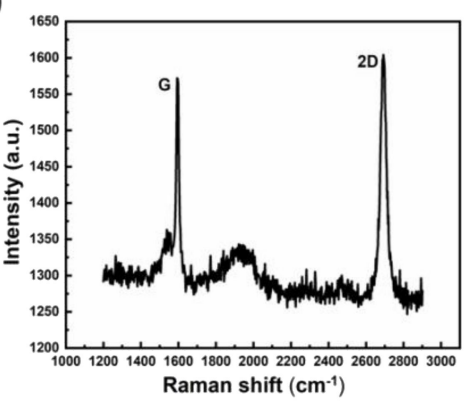

Figure 7. (a) Optical micrograph of single-crystal graphene on the back of the copper foil under 100-times magnification. (b) SEM image of single-crystal graphene domains on the copper foil. (c) Optical micrograph of single-crystal graphene transferred onto silicon dioxide grown on Si wafers under 100-times magnification. (d) The Raman spectra of the single-crystal graphene transferred onto the silicon dioxide grown on Si wafers.

\section{Conclusions}

In conclusion, the nucleation of graphene at different locations in the quartz boat was explored by setting up several comparative experiments. We found that the nucleation density of graphene at the bottom is lower than that at the top, and that at the location near the gas inlet side is lower than that near the gas outlet in the quartz boat. We also found that the single-crystal graphene can grow on the back of outer surfaces of copper pockets (the side facing the bottom of the quartz boat). The single-crystal graphene with a diameter of $800 \mu \mathrm{m}$ can be obtained directly on copper foils by our method after growing for $60 \mathrm{~min}$. Raman spectroscopy revealed the single-crystal graphene to be in uniform monolayers with a low D-band intensity. The single-crystal graphene prepared in our work can be used in many kinds of 2D electronics, such as flexible electrodes and flexible field effect transistors, because of its ultra-high carrier mobility and excellent flexibility.

Author Contributions: Conceptualization: B.Z. and K.Y.; Methodology: K.Y.; Software: K.Y.; Validation: R.J., J.L. and F.C.; Formal analysis: K.Y.; Investigation: K.Y., J.L. and R.J.; Resources: B.Z., L.L. and Q.G.; Data curation: K.Y. and R.J.; Writing—original draft preparation: K.Y.; Writing—review and editing: B.Z., L.L. and J.L.; Visualization: K.Y.; Supervision: Z.Y., F.C. and Y.G.; Project administration: B.Z., L.L. and J.Y.; Funding acquisition: B.Z., L.L. and Z.Y. All authors have read and agreed to the published version of the manuscript.

Funding: This research was funded by the National Natural Science Foundation of China (No.61571103, 62075035), the Guangdong Basic and Applied Basic Research Foundation (2021B1515420001), the Innovation Team of Colleges and Universities in Guangdong Province (2020KCXTD030 and 2018KCXTD033), the Science and Technology Project of Zhongshan (No.2019B2018), and the Science and Technology Foundation of Guangdong Province (No.2021A0101180005). 
Conflicts of Interest: All authors have seen and approved the final version of the manuscript being submitted. The authors confirm that the article is their original work, and it has not received prior publication and is not under consideration for publication elsewhere.

\section{References}

1. Novoselov, K.S.; Geim, A.K.; Morozov, S.V.; Jiang, D.; Zhang, Y.; Dubonos, I.; Grigorieva, I.V.; Firsov, A.A. Electric Field Effect in Atomically Thin Carbon Film. Science 2004, 306, 666. [CrossRef]

2. Novoselov, K.S.; Geim, A.K.; Morozov, S.V.; Jiang, D.; Katsnelson, M.I.; Grigorieva, I.V.; Dubonos, I.; Firsov, A.A. Two-dimensional gas of massless Dirac fermions in graphene. Nature 2005, 438, 197-200. [CrossRef] [PubMed]

3. Zhao, G.; Li, X.; Huang, M.; Zhen, Z.; Zhong, Y.; Chen, Q.; Zhao, X.; He, Y.; Hu, R.; Yang, T.; et al. The physics and chemistry of graphene-on-surfaces. Chem. Soc. Rev. 2017, 46, 4417-4449. [CrossRef]

4. Banszerus, L.; Schmitz, M.; Engels, S.; Dauber, J.; Oellers, M.; Haupt, F.; Watanabe, K.; Taniguchi, T.; Beschoten, B.; Stampfer, C. Ultrahigh-mobility graphene devices from chemical vapor deposition on reusable copper. Sci. Adv. 2015, 1, e1500222. [CrossRef] [PubMed]

5. Jang, H.; Park, Y.J.; Chen, X.; Das, T.; Kim, M.S.; Ahn, J.H. Graphene-Based Flexible and Stretchable Electronics. Adv. Mater. 2016, 28, 4184-4202. [CrossRef] [PubMed]

6. Novoselov, K.S.; Fal'Ko, V.I.; Colombo, L.; Gellert, P.R.; Schwab, M.G.; Kim, K. A roadmap for graphene. Nature 2012, 490, 192-200. [CrossRef] [PubMed]

7. Fatemi, S.M.; Abbasi, Z.; Rajabzadeh, H.; Hashemizadeh, S.A.; Deldar, A.N. A review of recent advances in molecular simulation of graphene-derived membranes for gas separation. Eur. Phys. J. D 2017, 71, 194. [CrossRef]

8. Zhang, J.; Lin, L.; Sun, L.; Huang, Y.; Koh, A.L.; Dang, W.; Yin, J.; Wang, M.; Tan, C.; Li, T.; et al. Clean Transfer of Large Graphene Single Crystals for High-Intactness Suspended Membranes and Liquid Cells. Adv. Mater. 2017, 29, 1-7. [CrossRef]

9. Wang, C.; Chen, W.; Han, C.; Wang, G.; Tang, B.; Tang, C.; Wang, Y.; Zou, W.; Chen, W.; Zhang, X.; et al. Growth of millimeter-size single crystal graphene on $\mathrm{Cu}$ foils by circumfluence chemical vapor deposition. Sci. Rep. 2014, 4, 4537. [CrossRef]

10. Bae, S.; Kim, H.; Lee, Y.; Xu, X.; Park, J.S.; Zheng, Y.; Balakrishnan, J.; Lei, T.; Kim, H.R.; Song, Y.I.; et al. Roll-to-roll production of 30-inch graphene films for transparent electrodes. Nature Nanotech. 2010, 5, 574-578. [CrossRef]

11. Wei, D.; Liu, Y. Controllable synthesis of graphene and its applications. Adv. Mater. 2010, 22, 3225-3241. [CrossRef] [PubMed]

12. Yang, X.; Zhang, G.; Prakash, J.; Chen, Z.; Gauthier, M.; Sun, S. Chemical vapour deposition of graphene: Layer control, the transfer process, characterisation, and related applications. Int. Rev. Phys. Chem. 2019, 38, 149-199. [CrossRef]

13. Yakobson, B.I.; Ding, F. Observational geology of graphene, at the nanoscale. ACS Nano 2011, 5, 1569-1574. [CrossRef] [PubMed]

14. Wang, D.; Zhang, Z.H.; Deng, X.Q.; Fan, Z.Q.; Tang, G.P. Magnetism and magnetic transport properties of the polycrystalline graphene nanoribbon heterojunctions. Carbon 2016, 98, 204-212. [CrossRef]

15. Lee, W.; Kihm, K.D.; Kim, H.G.; Shin, S.; Lee, C.; Park, J.S.; Cheon, S.; Kwon O., M.; Lim, G.; Lee, W. In-Plane Thermal Conductivity of Polycrystalline Chemical Vapor Deposition Graphene with Controlled Grain Sizes. Nano Lett. 2017, 17, 2361-2366. [CrossRef] [PubMed]

16. Yazyev, O.V.; Chen, Y.P. Polycrystalline graphene and other two-dimensional materials. Nat. Nanotechnol. 2014, 9, 755-767. [CrossRef]

17. Duong, D.L.; Han, G.H.; Lee, S.M.; Gunes, F.; Kim, E.S.; Kim, S.T.; Kim, H.; Ta, Q.H.; So, K.P.; Yoon S., J.; et al. Probing graphene grain boundaries with optical microscopy. Nature 2012, 490, 235-239. [CrossRef]

18. Ma, T.; Liu, Z.; Wen, J.; Gao, Y.; Ren, X.; Chen, H.; Jin, C.; Ma, X.; Xu, N.; Cheng, H.; et al. Tailoring the thermal and electrical transport properties of graphene films by grain size engineering. Nat. Commun. 2017, 8, 14486. [CrossRef] [PubMed]

19. Yu, Q.; Jauregui, L.A.; Wu, W.; Colby, R.; Tian, J.; Su, Z.; Cao, H.; Liu, Z.; Pandey, D.; Wei, D.; et al. Control and characterization of individual grains and grain boundaries in graphene grown by chemical vapour deposition. Nat. Mater. 2011, 10, 443-449. [CrossRef]

20. Zhang, J.; Lin, L.; Jia, K.; Sun, L.; Peng, H.; Liu, Z. Controlled Growth of Single-Crystal Graphene Films. Adv. Mater. 2020, 32, 1-27. [CrossRef]

21. Li, X.; Magnuson, C.W.; Venugopal, A.; Tromp, R.M.; Hannon, J.B.; Vogel, E.M.; Colombo, L.; Ruoff, R.S. Large-area graphene single crystals grown by low-pressure chemical vapor deposition of methane on copper. J. Am. Chem. Soc. 2011, 133, 2816-2819. [CrossRef] [PubMed]

22. Iwasaki, T.; Park, H.J.; Konuma, M.; Lee, D.S.; Smet, J.H.; Starke, U. Long-range ordered single-crystal graphene on high-quality heteroepitaxial $\mathrm{Ni}$ thin films grown on $\mathrm{MgO}(111)$. Nano Lett. 2011, 11, 79-84. [CrossRef] [PubMed]

23. Wofford, J.M.; Starodub, E.; Walter, A.L.; Nie, S.; Bostwick, A.; Bartelt, N.C.; Thümer, K.; Rotenberg, E.; McCarty, K.F.; Dubon, O.D. Extraordinary epitaxial alignment of graphene islands on $\mathrm{Au}(111)$. New J. Phys. 2012, 14, 053008. [CrossRef]

24. Gao, M.; Pan, Y.; Huang, L.; Hu, H.; Zhang, L.Z.; Guo, H.M.; Du, S.X.; Gao, H.J. Epitaxial growth and structural property of graphene on $\mathrm{Pt}(111)$. Appl. Phys. Lett. 2011, 98, 2009-2012. [CrossRef]

25. Deng, B.; Xin, Z.; Xue, R.; Zhang, S.; Xu, X.; Gao, J.; Tang, J.; Qi, Y.; Wang, Y.; Zhao, Y.; et al. Scalable and ultrafast epitaxial growth of single-crystal graphene wafers for electrically tunable liquid-crystal microlens arrays. Sci. Bull. 2019, 64, 659-668. [CrossRef]

26. Wu, T.; Zhang, X.; Yuan, Q.; Xue, J.; Lu, G.; Liu, Z.; Wang, H.; Wang, H.; Ding, F.; Yu, Q.; et al. Fast growth of inch-sized single-crystalline graphene from a controlled single nucleus on Cu-Ni alloys. Nat. Mater. 2016, 15, 43-47. [CrossRef] [PubMed] 
27. Ago, H.; Ohta, Y.; Hibino, H.; Yoshimura, D.; Takizawa, R.; Uchida, Y.; Tsuji, M.; Okajima, T.; Mitani, H.; Mizuno, S. Growth Dynamics of Single-Layer Graphene on Epitaxial Cu Surfaces. Chem. Mat. 2015, 27, 5377-5385. [CrossRef]

28. Brown, L.; Lochocki, E.B.; Avila, J.; Kim, C.J.; Ogawa, Y.; Havener, R.W.; Kim, D.; Monkman, E.J.; Shai, D.E.; Wei, H.I.; et al. Polycrystalline graphene with single crystalline electronic structure. Nano Lett. 2014, 14, 5706-5711. [CrossRef]

29. Hu, B.; Ago, H.; Ito, Y.; Kawahara, K.; Tsuji, M.; Magome, E.; Sumitani, K.; Mizuta, N.; Ikeda, K.; Mizuno, S. Epitaxial growth of large-area single-layer graphene over $\mathrm{Cu}(111)$ /sapphire by atmospheric pressure CVD. Carbon 2012, 50, 57-65. [CrossRef]

30. Hao, Y.; Wang, L.; Liu, Y.; Chen, H.; Wang, X.; Tan, C.; Nie, S.; Suk, J.W.; Jiang, T.; Liang, T.; et al. Oxygen-activated growth and bandgap tunability of large single-crystal bilayer graphene. Nat. Nanotechnol. 2016, 11, 426-431. [CrossRef]

31. Chen, S.; Brown, L.; Levendorf, M.; Cai, W.; Ju, S.Y.; Edgeworth, J.; Li, X.; Magnuson, C.; Velamakanni, A.; Piner, R.R.; et al. Oxidation resistance of graphene-coated $\mathrm{Cu}$ and $\mathrm{Cu} / \mathrm{Ni}$ alloy. ACS Nano 2011, 5, 1321-1327. [CrossRef]

32. Chen, S.; Ji, H.; Chou, H.; Li, Q.; Li, H.; Suk, J.W.; Piner, R.; Liao, L.; Cai, W.; Ruoff, R.S. Millimeter-Size Single-Crystal Graphene by Suppressing Evaporative Loss of Cu During Low Pressure Chemical Vapor Deposition. Adv. Mater. 2013, 25, $2062-2065$. [CrossRef] [PubMed] 